\title{
A Highly Efficient Thermally Controlled Loss-Tunable Long-Period Fiber Grating on Corrugated Metal Substrate
}

\author{
Enboa Wu, Rou-Ching Yang, Kuo-Ching San, Chien-Hung Lin, Fares Alhassen, and H. P. Lee
}

\begin{abstract}
We demonstrate a new approach for fabricating a compact loss-tunable long-period fiber grating (LPFG) by gluing a fiber onto a corrugated metal substrate filled with ultraviolet-cured epoxy. The strain caused by the difference in thermal expansion among the adhesive, the substrate, and the silica fiber induces a periodic microbending along the fiber when the device temperature is changed. The LPFG has an initial flat spectrum at room temperature. With 35 grating periods, a tuning range of $-21 \mathrm{~dB}$ is achieved through core-cladding coupling for a temperature change from room temperature to $4^{\circ} \mathrm{C}$. A theoretical model is presented to explain the high tuning efficiency and the operating mechanisms of the device.
\end{abstract}

Index Terms-Long-period fiber grating (LPFG), loss tunable filter, thermally tuned fiber device.

\section{INTRODUCTION}

$\mathbf{L}$ ONG-PERIOD fiber gratings (LPFGs) which couple light from the core mode to cladding modes have been widely studied for applications in fiber-optic communications such as band rejection filters [1], mode converters [2], spectral equalizers [3], and variable optical attenuators [4]. Their high sensitivity to temperature and strain (bending, tension, and torsion) has led to the development of a multitude of LPFG-based sensors [5], [6]. Because of their low insertion loss and return loss, LPFGs can be concatenated to realize complex dynamic filtering profiles. For example, wavelength-tunable and loss-tunable broad-band LPFGs have been investigated for dynamic gain flattening filters in erbium-doped fiber amplifiers [4] and cascaded two-section LPFGs have been studied for sensor applications [5]. A strain-induced thermally controlled loss-tunable LPFG was demonstrated using etched fiber on a corrugated Si substrate [7], [8]. However, the device has a low tuning efficiency (a temperature difference of $250{ }^{\circ} \mathrm{C}$ is required to achieve a $20-\mathrm{dB}$ tuning range) and a temperature-dependent wavelength shift. In addition, the inability to control the filtering depth at its off state (room temperature) implies that a large amount of power is required for its operation.

Manuscript received August 31, 2004; revised October 22, 2004. This work was supported by the National Science Council of the Republic of China under Contract NSC93-2218-E-002-053.

E. Wu, R.-C. Yang, and K.-C. San are with the Institute of Applied Mechanics, College of Engineering, National Taiwan University, Taipei 106, Taiwan. R.O.C. (e-mail: ebwu@iam.ntu.edu.tw).

C.-H. Lin, F. Alhassen, and H. P. Lee are with the Department of Electrical Engineering and Computer Science, Henry Samueli School of Engineering, University of California, Irvine, CA 92697 USA.

Digital Object Identifier 10.1109/LPT.2004.842333

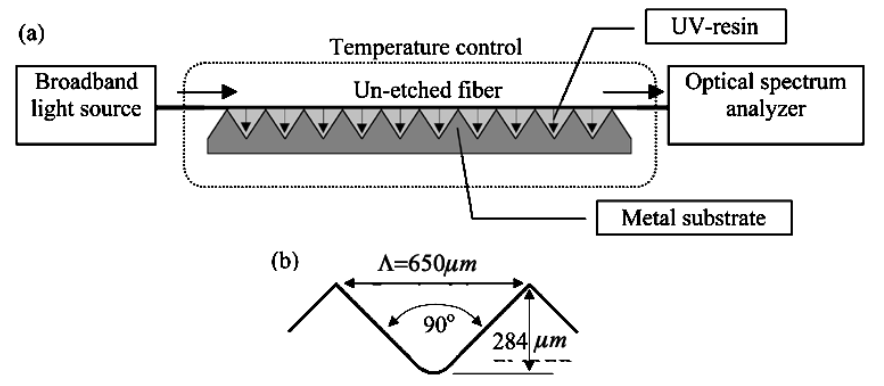

Fig. 1. (a) Schematic of the thermally tuned adhesive bonded LPFG, and (b) a detailed view of the corrugated V-groove.

We present a new approach to realize a temperature-controlled loss tunable LPFG by bonding a single-mode fiber (SMF) onto a corrugated metal substrate filled with ultraviolet (UV)-epoxy. The device shows a $>160$ fold improvement in tuning efficiency over the previous design [7], [8] and a near-zero insertion loss at its off state at room temperature. By drastically improving the temperature sensitivity of the LPFG, we have not only improved the power efficiency of the device but also narrowed down the temperature range needed for operating the device. These performance enhancements, together with the capability to adjust the filtering spectrum at the "off state" at room temperature, render this new design an attractive building block for a practical all-fiber dynamic spectral equalizer. The operation mechanisms behind the high tuning efficiency are explained in detail by a theoretical model.

\section{FABRICATION PROCESS AND Operating PRINCIPLE}

Fig. 1(a) shows the schematic of the LPFG device. A stepindex SMF (Model 310, Prime Optical Fiber Corp., core diameter of $9.1 \mu \mathrm{m}$ and $\Delta n / n$ of $0.30 \%$ ) is glued onto a corrugated stainless steel (S304) fixture using Chemitech U-425 UV-epoxy. The corrugated substrate is fabricated using the conventional high-precision wire-cutting method. The grating pitch is designed to be $650 \mu \mathrm{m}$, the depth and the angle of the $\mathrm{V}$-groove are $284 \mu \mathrm{m}$ and $90^{\circ}$ [Fig. 1(b)], respectively. Young's modulus and coefficient of thermal expansion (CTE) for stainless steel, fiber, and UV-epoxy below its glass transition temperature $\left(50{ }^{\circ} \mathrm{C}\right)$ are listed in Table I [9], [10]. After the corrugated V-grooves are filled up with UV-epoxy, the fiber is mounted on the tips of the corrugated grooves. Only the lower half of the fiber is immersed in the adhesive. The entire substrate is then cured by UV exposure at room temperature. After the curing process, the 
TABLE I

PHYSICAL PROPERTIES

\begin{tabular}{c|c|c|c}
\hline \hline Material & $\begin{array}{c}\text { Stainless } \\
\text { steel }\end{array}$ & Fiber & UV-epoxy \\
\hline $\begin{array}{c}\text { Young's Modulus } \\
(\mathrm{GPa})\end{array}$ & 193 & 70 & 2.3 \\
\hline $\mathrm{CTE}\left(\mathrm{ppm} /{ }^{\circ} \mathrm{C}\right)$ & 17.0 & 0.50 & 69.1 \\
\hline
\end{tabular}

fiber is permanently bonded to the substrate. A thermal electric cooler is used to control the device temperature.

The operation principle of the device can be understood qualitatively as follows: When the temperature is lowered, the contraction of the UV-epoxy forms a concave surface inside the $\mathrm{V}$-groove. Since the fiber is bonded to the adhesive, it is drawn toward the $\mathrm{V}$-grooves while being supported mechanically at the tips of the corrugated substrate [Fig. 1(a)]. Moreover, the larger contraction of the metal substrate relative to the fiber provides a net lateral compressive force on the fiber that further aids the downward bending of the fiber toward the $\mathrm{V}$-groove. The magnitude of the microbending, and hence, the loss amplitude of the LPFG can, therefore, be controlled by changing the device temperature. The transmission spectra of the LPFGs were measured using a broad-band superluminescent diode and an Anritsu 9710C optical spectrum analyzer. The period of the metal substrate was designed to satisfy the phase matching conditions for coupling the core mode to $\mathrm{LP}_{11}^{\mathrm{cl}}$ and $\mathrm{LP}_{12}^{\mathrm{cl}}$ cladding modes near 1530 and $1585 \mathrm{~nm}$, respectively.

\section{EXPERIMENTAL RESULTS AND ANALYSIS}

The transmission spectra for LPG 1 and LPG 2, with 28 and 35 grating periods, respectively, are shown in Fig. 2(a) and (b). In Fig. 2(a), the loss peaks for coupling to both $\mathrm{LP}_{11}^{\mathrm{cl}}$ and $\mathrm{LP}_{12}^{\mathrm{cl}}$ modes increase monotonically with decreasing temperature. The loss peak associated with $\mathrm{LP}_{12}^{\mathrm{cl}}$ mode coupling is larger than that of the $\mathrm{LP}_{11}^{\mathrm{cl}}$ mode due to a larger modal overlap for $\mathrm{LP}_{12}^{\mathrm{cl}}$ mode than for $\mathrm{LP}_{11}^{\mathrm{cl}}$ mode for a microbending-induced index perturbation [11]. In Fig. 2(b), the loss peak for $\mathrm{LP}_{12}^{\mathrm{cl}}$ mode reaches a maximum attenuation of $-21 \mathrm{~dB}$ at a temperature drop of $22{ }^{\circ} \mathrm{C}$. Further lowering of temperature results in a decrease of attenuation due to over-coupling. The enhanced coupling strength and narrower filtering bandwidth in LPG 2 is due to a longer coupling length in LPG 2 than LPG 1. Increasing the number of grating can further improve the temperature sensitivity [10]. Both LPFGs are initially off at room temperature, i.e., with zero insertion loss. By close investigation, detectable loss starts to be observed when the temperature drops below $20^{\circ} \mathrm{C}$.

Fig. 3(a) depicts the attenuation and wavelength shift versus ambient temperature in LPG 2. As the temperature is decreased from room temperature to $-15{ }^{\circ} \mathrm{C}$, it is clear that mode coupling to $\mathrm{LP}_{12}^{\mathrm{Cl}}$ mode becomes over-coupled below $4{ }^{\circ} \mathrm{C}$. As temperature is further reduced, the peak loss decreases due to over-coupling until it reaches $-13 \mathrm{~dB}$ at $-10{ }^{\circ} \mathrm{C}$, rather than fully recovering at $0 \mathrm{~dB}$. This interesting over-coupling phenomenon is different from that observed in the LPFGs induced by acoustic excitation, and is believed to be due to (a)

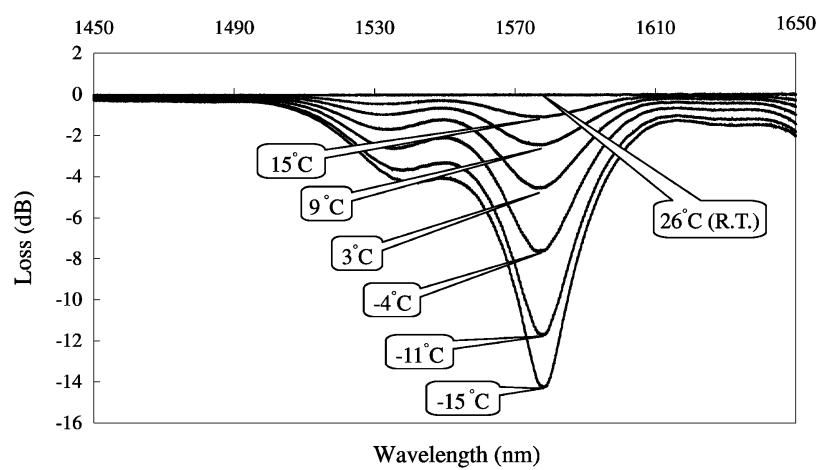

(b)

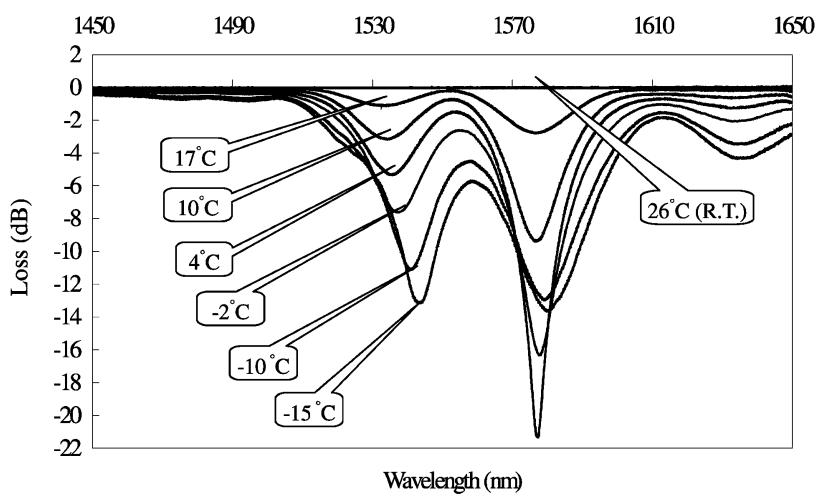

Fig. 2. Transmission spectra of LPFG versus cooling temperature for (a) LPG $1(N=28)$. (b) LPG $2(N=35)$, over-coupling phenomenon for $\mathrm{LP}_{12}^{\mathrm{cl}}$ mode is clearly evident.

(a)

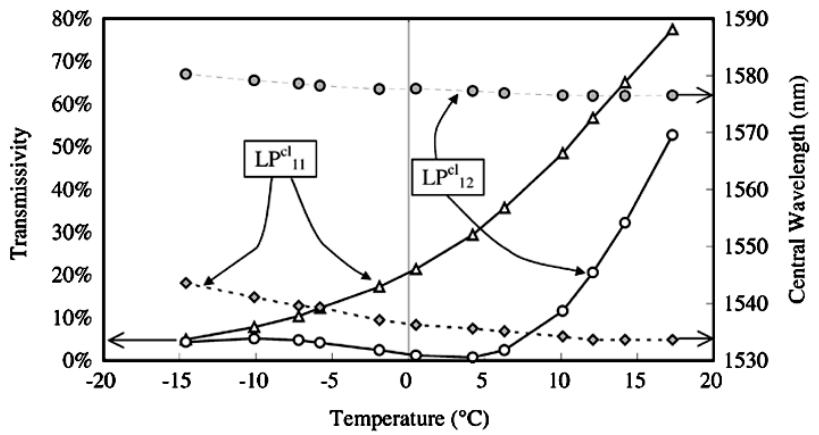

(b)

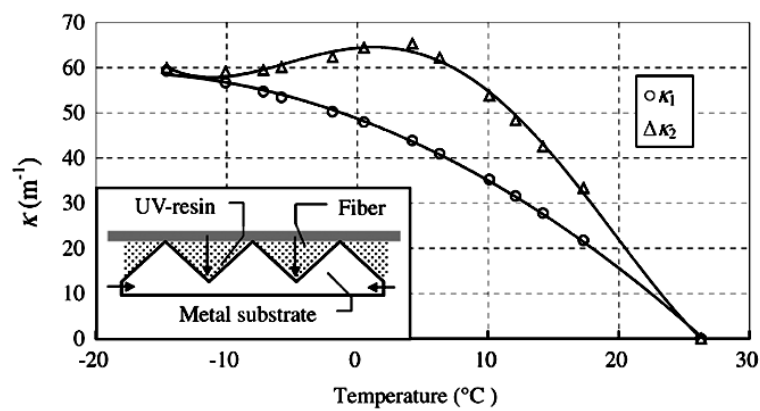

Fig. 3. For LPG $2(N=35)$, (a) a plot for attenuation and wavelength shift versus cooling temperature for LPG 2, and (b) a plot for coupling coefficient versus cooling temperature for LPG 2. $\kappa_{1}$ and $\kappa_{2}$ are the coupling coefficients for core mode coupling to $\mathrm{LP}_{11}^{\mathrm{cl}}$ and $\mathrm{LP}_{12}^{\mathrm{cl}}$ modes, respectively. The pushing forces from the substrate and the pulling forces from the UV-epoxy bend the fiber segment as illustrated in the inset of (b).

the radiation loss of the cladding mode in contact with the epoxy, which has an index close to 1.5 . Only the $\mathrm{LP}_{12}^{\mathrm{cl}}$ mode coupling undergoes an over-coupling transition; the $\mathrm{LP}_{11}^{\mathrm{cl}}$ mode coupling 
monotonically increases throughout the $41{ }^{\circ} \mathrm{C}$ decrease in temperature. This is consistent with the fact that the $\mathrm{LP}_{11}^{\mathrm{cl}}$ mode is more tightly confined to the fiber than the $\mathrm{LP}_{12}^{\mathrm{cl}}$ mode, and consequently incurs a smaller radiation loss. The efficiency of LPG 2 is studied by plotting the temperature versus the coupling coefficients $\kappa_{i}, i=1,2$ relationship, as shown in Fig. 3(b), where $\kappa_{1}$ and $\kappa_{2}$ are the coupling coefficients for core mode coupling to $\mathrm{LP}_{11}^{\mathrm{cl}}$ and $\mathrm{LP}_{12}^{\mathrm{cl}}$ modes, respectively [12]. The change of coupling coefficient per temperature $d \kappa_{i} / d T$ near $\kappa_{i}=0$ is $-0.0257 / \mathrm{cm} \cdot{ }^{\circ} \mathrm{C}$ for $\mathrm{LP}_{11}^{\mathrm{cl}}$ mode and $-0.0611 / \mathrm{cm} \cdot{ }^{\circ} \mathrm{C}$ for $\mathrm{LP}_{12}^{\mathrm{cl}}$ mode, respectively. These two $d \kappa_{i} / d T$ values remain relatively constant as temperature is lowered from room temperature and then decreased progressively to zero as the LPFG approaches the over-coupling state. Nevertheless, these values near $\kappa=0$ are an order of magnitude larger than those of the previous design [7], [8]. Taking into account the use of etched fiber in the previous device, which lead to a nearly order of magnitude reduction in bending rigidity and a two-fold increase in modal overlap, the actual enhancement of tuning efficiency per unit temperature change in the present design is $>160$ times higher than that of the previous design.

This two order of magnitude enhancement in efficiency is due to multiple innovations in the mechanical design. When the device is cooled, each fiber segment within a V-groove is pulled downward by thermal contraction of the UV-epoxy and pushed horizontally by the contraction of metallic substrate, as shown in the inset of Fig. 3(b), due to differential contractions between the epoxy and the fiber, and between substrate and fiber. In contrast, in the device in [7] and [8], the etched fiber was bent by a thin layer of temperature-cured epoxy glued to only a small portion of the fiber segment into the grooves of a Si substrate. The present design also offers a much larger contact area between the fiber and the epoxy, and hence, a stronger pulling force and a mechanically more robust device.

The deflection of each fiber segment in this device can be modeled using the Euler-Bernoulli beam theory. The governing equation as derived as [13] is

$$
E I w^{\prime \prime \prime \prime}+P w^{\prime \prime}=q(x)
$$

The four boundary conditions of (1) are

$$
\begin{array}{ll}
w(x)=w^{\prime}(x)=0, & \text { at } x=0 \\
w(x)=w^{\prime}(x)=0, & \text { at } x=l
\end{array}
$$

i.e., the deflection and the slope are zero at these two ends. In (1), $E$ is the Young's modulus and $I$ is the moment of inertia of the fiber $\left(I=1.2 \times 10^{-17} \mathrm{~m}^{4}\right), l$ is the pitch of each grating $(l=$ $650 \mu \mathrm{m}), w(x)$ is the fiber deflection profile in each segment of grating, $P$ is the axial compressive force caused from the substrate contraction, and $q(x)$ is the distributive pulling force due to the contraction of the epoxy. Because the problem is nonlinear in nature, the value for $w(x)$ in a sinusoidal profile was calculated by iteration. A linear relationship between the maximum deflection and the ambient temperature is obtained for a temperature range from $26^{\circ} \mathrm{C}$ down to $-15^{\circ} \mathrm{C}$ as $w_{\max }=-7.36 \times \Delta T$ $\left({ }^{\circ} \mathrm{C}\right)(\mathrm{nm})$, where $\Delta T\left({ }^{\circ} \mathrm{C}\right)$ is the temperature difference from the room temperature, $26^{\circ} \mathrm{C}$. Based on this calculation, when the temperature drops to $4{ }^{\circ} \mathrm{C}$, at which over-coupling starts to occur, the deflection of the fiber is $162 \mathrm{~nm}$. The contributions from the pulling by the UV-epoxy and the pushing by the metal substrate to the microbending of each fiber segment are $87 \%$ and $13 \%$, respectively, in the current design. It means that the pulling from the epoxy is the dominant mechanism for each fiber segment to bend. The analysis also shows that $w_{\max }$ and, thus, the tuning efficiency can be further enhanced by selecting a substrate with a smaller CTE and an epoxy with a larger CTE and Young's modulus.

\section{SUMMARY}

We have fabricated an efficient loss-tunable LPFG by gluing an unetched SMF onto a corrugated metal substrate using UV-cured epoxy. Using a relatively short LPFG, 35 grating periods and $22.75 \mathrm{~mm}$ long, we achieved $-21 \mathrm{~dB}$ at $4{ }^{\circ} \mathrm{C}$ through a temperature change of only $22{ }^{\circ} \mathrm{C}$. The cause of the high efficiency of this device is due to the combination of the thermal contractions of substrate in the horizontal direction and the UV-epoxy in the vertical direction when temperature is decreased. A theoretical model has been developed by which a linear relationship between the maximum deflection of each fiber segment and the temperature change of the device was obtained. The model also indicated that a more efficient LPFG can be designed by a proper selection of the materials made of the substrate and the adhesive.

\section{REFERENCES}

[1] A. M. Vengsarkar, P. J. Lemaire, J. B. Judkins, V. Bhatia, T. Erdogan, and J. E. Sipe, "Long-period fiber gratings as band-rejection filters," $J$. Lightw. Technol., vol. 14, no. 1, pp. 58-65, Jan. 1996.

[2] D. Ostling and H. E. Engan, "Broadband spatial mode conversion by chirped fiber bending," Opt. Lett., vol. 21, pp. 192-194, 1996.

[3] J. K. Bae, S. H. Kim, J. H. Kim, J. Bae, S. B. Lee, and J. M. Jeong, "Spectral shape tunable band-rejection filter using a long-period fiber grating with divided coil heaters," IEEE Photon. Technol. Lett., vol. 15, no. 3, pp. 407-409, Mar. 2003.

[4] S. Ramachandran, M. F. Yan, E. Monberg, F. V. Dimarello, P. Wisk, and S. Ghalmi, "Record bandwith, spectral flat coupling with microbend grating in dispersion tailored fiber," IEEE Photon. Technol. Lett., vol. 15, no. 11, pp. 1561-1563, Nov. 2003.

[5] V. Bhatta and A. M. Vengsarkar, "Optical fiber long-period grating sensors,” Opt. Lett., vol. 21, pp. 692-694, 1996.

[6] C. Y. Lin and L. A. Wang, "Loss-tunable long period fiber grating made from etched corrugation structure," Electron. Lett., vol. 35, no. 31, pp. 1872-1873, 1999.

[7] Y. Jiang, Q. Li, C. H. Lin, Ed. Lyons, I. Tomov, and H. P. Lee, "A novel strained-induced thermally tuned long-period fiber grating fabricated on a periodic corrugated silicon fixture," IEEE Photon. Technol. Lett., vol. 14, no. 7, pp. 941-943, Jul. 2002.

[8] C. H. Lin, Q. Li, A. A. Au, Y. Jiang, E. Wu, and H. P. Lee, "Straininduced thermally long-period fiber gratings fabricated on a periodically corrugated substrate," J. Lightw. Technol., vol. 22, no. 7, pp. 1818-1827, Jul. 2004.

[9] R. C. Hibbeler, Mechanics of Materials. Englewood Cliffs, NJ: Prentice-Hall, 2000.

[10] R. C. Yang, "Design, experiment, and analysis on tunable long period fiber grating," Master thesis (in Chinese), Inst. Appl. Mechanics, Nat. Taiwan Univ., 2004.

[11] Q. Li, X. Liu, J. Peng, B. Zhou, E. R. Lyons, and H. P. Lee, "Highly efficient acoustooptic tunable filter based on cladding etched single-mode fiber," IEEE Photon. Technol. Lett., vol. 14, no. 3, pp. 337-339, Mar. 2002.

[12] C. H. Lin, Q. Li, and H. P. Lee, "Periodic microbending-induced core-tocladding mode coupling in polarization-maintaining fibers," Opt. Lett., vol. 28, no. 12, pp. 998-1000, 2003.

[13] W. F. Chen and T. Atsuta, Theory of Beam Columns. New York: McGraw-Hill, 1976. 